\title{
Implementation of WiMAX (IEEE802.16.d) OFDM Baseband Transceiver-Based Multiwavelet OFDM on a Multi-Core Software-Defined Radio Platform
}

\author{
Mohammed Aboud kadhim and Widad Ismail \\ School of Electrical and Electronics Engineering, Universiti Sains Malaysia Seri Ampangan, 14300 Nibong Tebal, \\ Seberang Perai Selatan, Pulau Pinang, Malaysia \\ Correspondence should be addressed to Mohammed Aboud kadhim, makaboud@gmail.com
}

Received 23 January 2011; Accepted 19 February 2011

Academic Editors: L.-M. Cheng and M. A. Nappi

Copyright (C 2011 M. Aboud kadhim and W. Ismail. This is an open access article distributed under the Creative Commons Attribution License, which permits unrestricted use, distribution, and reproduction in any medium, provided the original work is properly cited.

\begin{abstract}
This paper investigates a new approach to the adaptation of the WiMAX IEEE802.16d baseband, the physical layer performance of wireless communications systems based on OFDM multiwavelet transform, using half values of coding rates, 16-QAM, and DMWT-OFDM by being applied to the SFF SDR development platform. In the new structure of WiMAX IEEE802.16d baseband, is reduce further the level of interference, and spectral efficiency is increased. The proposed design was model tested, and its performance was found to comply with International Telecommunications Union channel (ITU) models that have been elected for the wireless channel in the simulation process. The simulation approved the proposed design which achieved much lower bit error rates, increased signal-to-noise power ratio (SNR), robustness for multipath channels and does not require cyclically prefixed guard interval and have higher spectral efficiency than OFDM based on DWT and FFT also can be used as an alternative conventional OFDM in WiMAX IEEE802.16d baseband.
\end{abstract}

\section{Introduction}

Over the recent years, there has been important and growing demand for greater bandwidth. The problem with the existing transmission media similar fiber, coaxial and twisted pair is costly and complex to layout in rural areas. A solution to this problem is given by IEEE standard 802.16, famous as WiMax. WiMax could possibly erase the suburban and rural blackout areas that currently have no broadband internet access. WiMax short for "Worldwide Interoperability for microwave access" is the broadband internet technology which is growing quickly across the world. The frequency spectrum for WiMax has given by the IEEE is $2-11 \mathrm{GHz}$. The wireless communications industry is gaining momentum in both fixed and mobile applications. The nonstop increase in demand for all types of wireless services (voice, data, and multimedia) is fueling the require for higher capacity and data rates not only in fixed but too in mobile applications. WLANs and $3 \mathrm{G}$ cellular networks are undergo several problems for obtaining a complete mobile broadband access, bounded by factors such as bandwidth, coverage area, or structure costs. In this context, WiMAX appears to accomplish these requirements, providing vehicular mobility and high service areas and data rates. Defined to provide broadband wireless access, it is increasingly gaining concern as an alternative lastmile technology to DSL lines and cable modems, and a complete technology where wireless networks are not enough developed. IEEE 802.16d was developed for WiMAX wireless communication, which is based on orthogonal frequency-division multiplexing (OFDM) technology, to enable advancement towards $4 \mathrm{G}$. However, in wireless communication reception, the reliability of OFDM is limited because of the time-varying nature of the channel. This induces intercarrier interference (ICI) and increases inaccuracies in channel tracking. This can be avoided effectively at the cost of power loss and bandwidth expansion by inserting a cyclic prefix guard interval before each block 
of parallel data symbols. yet, the spectral efficiency of the OFDM system this guard interval is decreases in a corresponding amount. The implementations in practice of OFDM in WiMAX technology today have been done by using FFT and its inverse operation IFFT [1-4] Recently, based on Haar-orthonormal wavelets, discrete wavelet-based OFDM (DWT-OFDM) was found to reduce the intersymbol interference (ISI) and ICI, which are caused by the loss in orthogonality between the carriers. DWT-OFDM be able to too support much higher spectrum efficiency than discrete Fourier-based OFDM (DFT-OFDM) to represent data modulation and demodulation the concept of scalar wavelets has been exploited as wavelet modulation, for multi rate transmissions [5-9]. The Fourier-based OFDM uses complex exponential bases functions, and was replaced with orthonormal wavelets to reduce the level of interference. It was found that Haar-based orthonormal wavelets were capable of reducing the ISI and ICI, which were caused by the loss in orthogonality between the carriers. In [10] the simulation results showed the BER performance of OFDM system with different orthogonal bases (i.e., Fourierbased OFDM and wavelet-based OFDM). The simulations were found to have a great deal of channel dependence in the performance of wavelet and Fourier filters. The main incitation for using wavelet-based OFDM is the superior spectral containment properties of wavelet filters over Fourier filters. It has been found that under certain channel conditions, wavelet OFDM outperformed Fourier OFDM. However, under other channels, the situation is reversed (e.g., the selective fading channel). First used the wavelet OFDM in WiMAX (IEEE802.16.d) Baseband Transceiver [11], from the results, we can see that $\mathrm{S} / \mathrm{N}$ measure can be successfully increased and achieves considerably lower bit error rates and performs better than FFT-OFDM when using the wavelet OFDM within a desired wavelet basis function. Thus, wavelet-based OFDM outperforms the conventional OFDM WiMAX-based FFT OFDM. Further performance gains can be made by looking into alternative orthogonal basis functions and finding a better transform rather than Fourier and wavelet transform, multiwavelet is a new concept has been proposed in recent years. Multiwavelets have several advantages compared to single wavelets. A single wavelet cannot possess all the properties of orthogonality, symmetry, short support, and vanishing moments at the same time, but a multiwavelet can [12], for all the priorities of multiwavelet, a natural thought is applying it in OFDM. The multiwavelet transform is a newer alternative to the wavelet transform. Multiwavelets are very like to wavelets but have some important differences. In particular, whereas wavelets have associated scaling $\varphi(t)$ and wavelet functions $\psi(t)$, multiwavelets have two or more scaling and wavelet functions. For notational convenience, the set of scaling functions be able to be written using the vector notation $\Phi(t)=\left[\begin{array}{llll}\varphi_{1}(t) & \varphi_{2}(t) & \cdots & \varphi_{r}(t)\end{array}\right]^{T}$, where $\Phi(t)$ is called the multiscaling function. The multiwavelet function is defined from the set of wavelet functions as $\Psi(t)=$ $\left[\begin{array}{llll}\psi_{1}(t) & \psi_{2}(t) & \cdots & \psi_{r}(t)\end{array}\right]^{T}$ When $r=1, \Psi(t)$ is called a scalar wavelet, or simply wavelet. as in basically $n$ can be arbitrarily large, the multiwavelets studied to date are primarily for $r=2$. The multiwavelet two scale equations resemble those for scalar wavelets [13]:

$$
\begin{aligned}
& \Phi(t)=\sqrt{2} \sum_{k=-\infty}^{\infty} H_{k} \Phi(2 t-k), \\
& \psi(t)=\sqrt{2} \sum_{k=-\infty}^{\infty} G_{k} \Phi(2 t-k) .
\end{aligned}
$$

However $H_{k}$ and $G_{k}$, and are matrix filters (i.e., $H_{k}$ and $G_{k}$ are $n \times n$ matrices instead of scalar values). The matrix elements in these filters provide further degrees of freedom than a traditional scalar wavelet. These extra degrees of freedom are able to be used to incorporate useful properties into the multiwavelet filters, such as orthogonality, symmetry, and a high order of approximation. The key is to figure out how to make the good use of these extra degrees of freedom. Multifilter construction methods are currently being developed to exploit these extra degrees of freedom. However, the multichannel nature of multiwavelets also means that the subband structure resulting from passing a signal through a multifilter bank is different. Sufficiently different, in fact, that established quantization methods do not perform as well with multiwavelets as they do with wavelets [14]. Wavelets can be delineated in the context of a multiresolution analysis. It is probable to have more than one scaling (and wavelet) function. This is the thought behind multiwavelets, which are a natural expansion of the wavelets. Multiwavelets are designed to be simultaneously symmetric, orthogonal, and have short supports with high approximation power, which cannot be achieved simultaneously for wavelet using only one scaling function. The aim is to increase the number of scaling functions to elevate the approximation power to more than one scaling function. In [15] a new OFDM system was being introduced, based on multifilters called multiwavelets. It has two or more lowpass and high-pass filters, the purpose of this multiplicity is to achieve more properties which cannot be combined in other transforms (Fourier and wavelet). A very significant multiwavelet filter is the GHM filter suggested by Geronimo, Hardian, and Massopust [16]. The GHM will be used in the OFDM system for error-rate minimization in OFDM for WiMAX technology. The GHM basis presents a combination of orthogonality, symmetry, and compact support, which cannot be achieved by any scalar wavelet basis. For GHM system $H_{k}$ are four scaling matrices $H_{0}, H_{1}, H_{2}$, and $H_{3}$ [12]:

$$
\begin{array}{cc}
H_{0}=\left[\begin{array}{cc}
\frac{3}{5 \sqrt{2}} & \frac{4}{5} \\
\frac{-1}{20} & \frac{-3}{10 \sqrt{2}}
\end{array}\right], & H_{1}=\left[\begin{array}{cc}
\frac{3}{5 \sqrt{2}} & 0 \\
\frac{9}{20} & \frac{1}{\sqrt{2}}
\end{array}\right], \\
H_{2}=\left[\begin{array}{cc}
0 & 0 \\
\frac{9}{20} & \frac{-3}{10 \sqrt{2}}
\end{array}\right], & H_{3}=\left[\begin{array}{cc}
0 & 0 \\
\frac{-1}{20} & 0
\end{array}\right] .
\end{array}
$$


Moreover, there are also four scaling wavelet matrices for GHM system $G_{0}, G_{1}, G_{2}$, and $G_{3}$ :

$$
\begin{array}{cc}
G_{0}=\left[\begin{array}{cc}
\frac{-1}{20} & \frac{-3}{10 \sqrt{2}} \\
\frac{1}{10 \sqrt{2}} & \frac{3}{10}
\end{array}\right], & G_{1}=\left[\begin{array}{cc}
\frac{9}{20} & \frac{-1}{\sqrt{2}} \\
\frac{-9}{10 \sqrt{2}} & 0
\end{array}\right], \\
G_{2}=\left[\begin{array}{cc}
\frac{9}{20} & \frac{-3}{10 \sqrt{2}} \\
\frac{9}{10 \sqrt{2}} & \frac{-3}{10}
\end{array}\right], & G_{3}=\left[\begin{array}{cc}
\frac{-1}{20} & 0 \\
\frac{-1}{10 \sqrt{2}} & 0
\end{array}\right] .
\end{array}
$$

The new proposed of OFDM based multiwavelet transform in WiMAX technology, can be used as an alternative to the conventional WiMAX (IEEE802.16d). In proposed OFDM system was introduced based on multifilters, called Multiwavelets, and has two or more low-pass and high-pass filters. The purpose of this multiplicity is to achieve more properties that other transforms (Fourier and wavelet) are unable to combine. The GHM is used in the OFDM system block.In the multiwavelet setting, GHM multiscaling and multiwavelet function coefficients are $2 \times 2$ matrices that must multiply vectors, instead of scalars during transformation step. This means that the multifilter bank needs two input rows. The goal of preprocessing is to relate the given scalar input signal of length $N$ to a sequence of length-2 vectors to start the analysis algorithm and reduce noise effects. In the one-dimensional signals, the computational method for DMWT and inverse DMWT by an oversampled scheme of preprocessing (repeated row), is convenient and influential and further performance gains were made by looking into alternative orthogonal bases functions and finding a better transform than Fourier and wavelet transforms. We determined this transform to be a discrete multiwavelets transform (DMWT). In this paper, the design of the OFDM of WiMAX systems based on DMWT, simulations results, and evaluation tests of these proposed systems are given. The results of both systems in the International Telecommunications Union (ITU) channel models will be examined and compared. In this paper the performance results of the simulated of WiMAX (IEEE802.16.d) based DMWT-OFDM, DWT-OFDM and DFT-OFDM were also compared among themselves.

\section{Proposed WiMAX (IEEE802.16.d) OFDM Based DMWT}

The block diagram in Figure 1 represents the whole system model or signal chain at the base band. This figure illustrates a typical OFDM system used for multicarrier modulation. The block system is divided into three main sections: the transmitter, receiver, and channel. Data are generated from a random source, and consist of a series of ones and zeros. Since the transmission is conducted block-wise, when forward error correction (FEC) is applied, the size of the data generated depends on the block size used. These data are converted into lower rate sequences via serial-to-parallel conversion and randomize it to avoid a long run of zeros or ones. The result is ease in carrier recovery at the receiver. The randomized data are encoded when the encoding process consists of a concatenation of an outer Reed-Solomon (RS) code. The implemented RS encoder is derived from a systematic RS Code using field generator GF $\left(2^{8}\right)$ and an inner convolutional code (CC) as an FEC scheme. This denotes that the first data pass in block format through the RS encoder, and goes across the convolutional encoder. It is a flexible coding process caused by the puncturing of the signal, and allows different coding rates. The last part of the encoder is a process of interleaving to prevent long error bursts using tail biting CCs with different coding rates (puncturing of codes is provided in the standard) [1]. Finally, interleaving is conducted using a two-stage permutation; the first aims to avoid the mapping of adjacent coded bits on adjacent subcarriers, while the second ensures that adjacent coded bits are mapped alternately onto relatively significant bits of the constellation, thus avoiding long runs of lowly reliable bits. The training frame (pilot subcarriers frame) is inserted and sent prior to the information frame. This pilot frame is used to create channel estimation used to compensate for the channel effects on the signal. The coded bits are then mapped to form symbols. The modulation scheme used is 16-QAM coding rate $(1 / 2)$ with gray coding in the constellation map. This process converts data to corresponding value of $\mathrm{M}$ ary constellation, which is a complex word (i.e., with a real and an imaginary part). The bandwidth $\left(B=\left(1 / T_{s}\right)\right)$ is divided into $N$ equally spaced subcarriers at frequencies $(k \Delta f), k=0,1,2, \ldots, N-1$ with $\Delta f=B / N$ and, $T_{s}$, the sampling interval. At the transmitter, information bits are grouped and mapped into complex symbols. In this system, (QAM) with constellation $C_{\mathrm{QAM}}$ is assumed for the symbol mapping [1]. Both the OFDM modulator and demodulator of the DMWT-based OFDM are shown in Figure 1. The training frame (pilot subcarriers frame) are inserted and sent prior to the information frame. This pilot frame is used to create channel estimation, which is used to compensate for the channel effects on the signal. To modulate spread data symbol on the orthogonal carriers, an N-point Inverse multiwavelet Transform IDMWT is used, as in conventional OFDM. Zeros are inserted in some bins of the IDMWT to compress the transmitted spectrum and reduce the adjacent carriers' interference. The added zeros to some subcarriers limit the bandwidth of the system, while the system without the zeros pad has a spectrum that is spread in frequency. The last case is unacceptable in communication systems, since one limitation of communication systems is the width of bandwidth. The addition of zeros to some subcarriers means not all the subcarriers are used; only the subset $\left(N_{c}\right)$ of total subcarriers $\left(N_{F}\right)$ is used. Hence, the number of bits in OFDM symbol is equal to $\log _{2}(M) * N_{c}$. Orthogonality between carriers is normally damaged when the transmitted signal is passed through a dispersive channel. When this occurs, the inverse transformation at the receiver cannot recover the data that was transmitted perfectly. Energy from one subchannel leaks into others, leading to interference. However, it is probable to rescue orthogonality by introducing a cyclic prefix (CP). This CP comprises of the final $v$ samples 


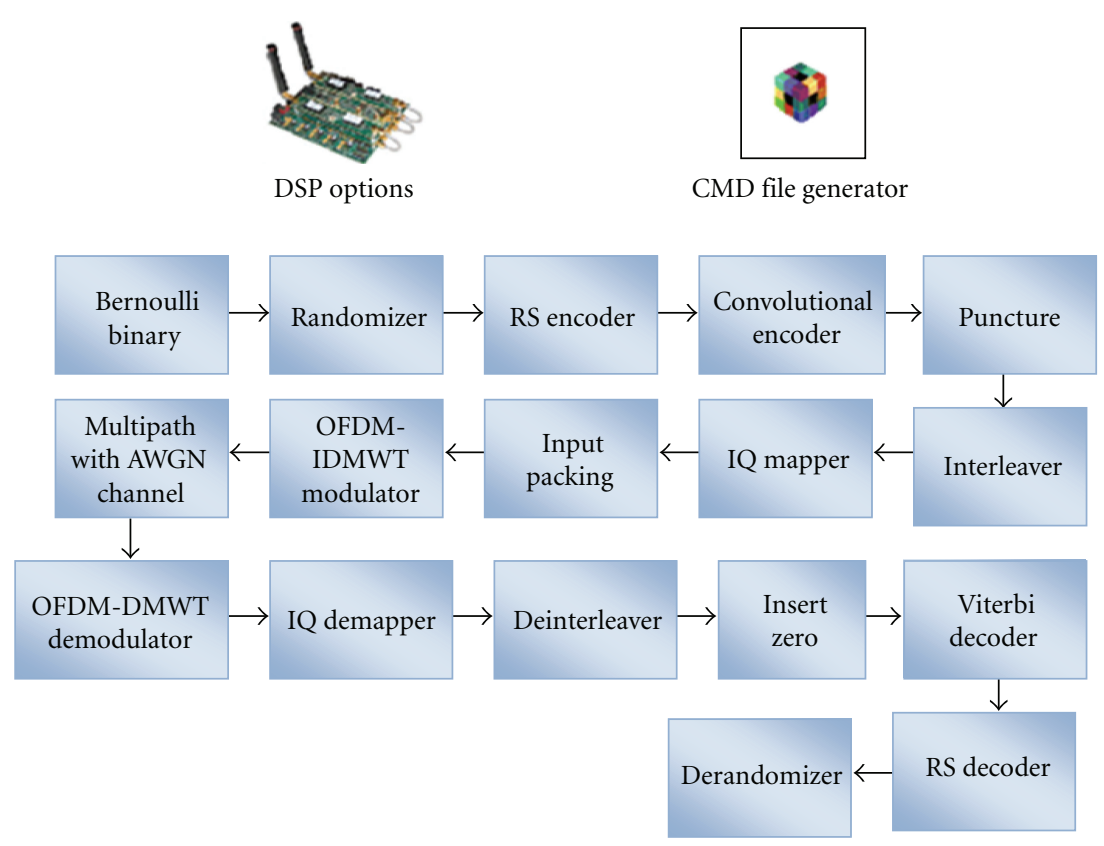

FIgURE 1: Block diagram of proposed IEEE802.16d WiMAX-based DMWT-OFDM.

of the original $K$ samples to be transmitted, prefixed to the transmitted symbol. The length $v$ is known by the channel's impulse response and is chosen to minimize ISI. If the impulse response of the channel has a length of less than or equal to $v$, the CP is sufficient to eliminate ISI and ICI. The efficiency of the transceiver is reduced by a factor of $K /(K+V)$; thus, it is desirable to make the $v$ as small or $K$ as large as possible. Therefore, the drawbacks of the CP are the loss of data throughput as precious bandwidth is wasted on repeated data. For this reason, finding another structure for OFDM to mitigate these drawbacks is necessary. If the number of subchannels is sufficiently large, the channel power spectral density can be assumed virtually flat within each subchannel. In these kinds of channels, multicarrier modulation has long been known to be optimum when the number of subchannels is large. The size of subchannels required $t$ approximate optimum performance depends on how rapidly the channel transfer function varies with frequency. The computation of DMWT and IDMWT 256 point is done by an oversampled scheme of preprocessing (repeated row), the procedure of computation in details in [15], which is explained in detail in the above section, is used. After which, the data converted from parallel to serial are fed to the channel proposed (ITU) models and the receiver performs the same operations as the transmitter, but in a reverse order, It further includes operations for synchronization and compensation for the destructive channel.

\section{SFF SDR Development Platforms}

The SFF SDR development platform shown in Figure 2 consists of three distinct hardware modules that offer flexible development capabilities: the digital processing, data conversion, and RF module. The digital processing module uses a Virtex-4 FPGA and a DM6446 SoC to offer developers the necessary performance for implementing custom IP and acceleration functions with varying requirements from one protocol to another supported on the same hardware. The data conversion module is equipped with dualchannel analog-to-digital and digital-to-analog converters. The RFmodule covers a variety of frequency ranges in transmission and reception, allowing it to support a wide range of applications [17].

3.1. System Performance Analysis and Optimization Target. MathWorks and Texas Instruments (TI), the two companies responsible for the development of MATLAB/Simulink, are currently working on the development of a DSP development tool that users can use through Simulink. The object modules, designed to meet their own needs, the programming system, which is implemented through RealTime Workshop, and the S-function with the TLC (Target Language Compiler) Function of the system design, when completed, can be directly converted to the most commonly used DSP programming language. The DSP, in conjunction with the TI software, Code Composer Studio, is completed 


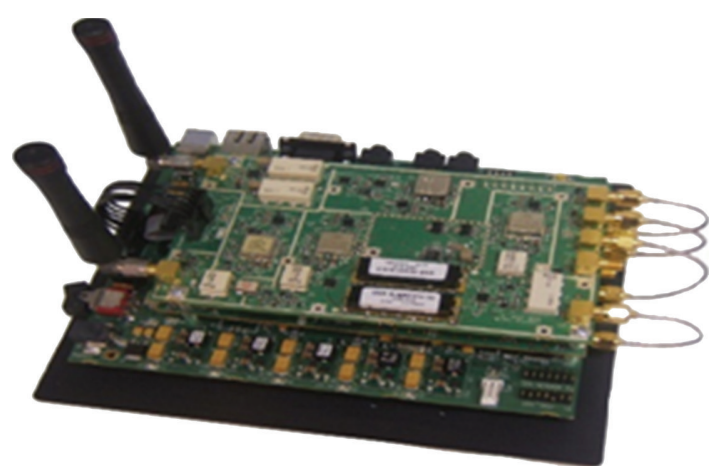

FIGURE 2: SFF SDR development platform [17].

in combination with the DSP hardware. Thus, through this development tool, users can work together to complete the design and simulation on the Simulink; however, it cannot provide the convenience of design that could increase the set count on the efficiency.

3.1.1. System Integration and Implementation of Workflow. In the development and testing of IEEE 802.16d wireless MANOFDM PHY, the specifications of communication transfer have varying systems, which are based on our needs under Simulink mentioned in the proposed system for DMWT based OFDM for WiMAX. For our study, we used the standard communication system box with a map provided by Matlab, which contains the following: internal communications blockset, signal processing blockset, and simulink blockset. These correspond to our use of the hardware development platform for SFF SDR DP Blockset. The overall WiMAX PHY system construction is opened in the Simulink interface and Matlab is used to communicate the internal functions of RTW and TLC. We intend to build a finished system into a module, in accordance with the code of each block. Through this, we can perform the compilation and completion that will be automatically compiled in Matlab CCS connecting knot. The CCS establishes a corresponding module under the file name "Project." We then correct the generated C code and conduct compilation, debugging, and analysis. We then download our work into to the DSP. The overall system workflow is shown in Figure 3. The figure shows the system built based on the Simulink-established IEEE 802.16d wireless MAN-OFDM PHY standard modules. The first step is the configuration by Simulink of the parameters interface and development platform into the conduct of the connecting node configuration. Information will be set to leave the bulk form of a fixed number of patterns, and the RTW system development module is set to be transferred and replaced by $\mathrm{C}$ language. Meanwhile, the TLC file option SDR development of modules and the set up Simulink system development are scheduled for DSP link module by an external module through the executive. Configuration of the IEEE 802.16d wireless MAN-OFDM PHY may be achieved through the DSP options block simulink to develop interfaces connecting node, development platform, and CCS. The use of the DSP options block and the compiler options
TABLE 1: System parameters.

\begin{tabular}{lc}
\hline Number of subcarriers & 256 \\
Number of FFT, DWT, DMWT points & 256 \\
Modulation type & $16-\mathrm{QAM}$ \\
Coding rate & $1 / 2$ \\
Channel bandwidth $B$ & $3.5 \mathrm{MHz}$ \\
Carrier frequency fc & $2.3 \mathrm{GHz}$ \\
MIMO fading correlations & $\rho_{T}=0.5, \rho_{R}=0.5$ \\
MIMO random phases & $\Phi_{1}=1.8, \Phi_{2}=2$, \\
Ncpc & $\Phi_{3}=0.23, \Phi_{4}=0.9$ \\
Ncbps & 4 \\
Number of data bits transmitted & 768 \\
\end{tabular}

allow us to optimize the system and the executive profit use. Moreover, future compiler optimization can be conducted through the Block. In the SFF SDR development platform of the DSP configuration, three kinds of memory are used: L1DRAM (8 KB), L2RAM (64 KB), and SDRAM (8 MB). The L1DRAM and L2RAM are used for the internal memory, while the SDRAM is used for the external memory. Due to the retention of internal memory, the speeds become quicker; thus, if information is to be placed in the internal memory in the system as a whole, the speeds and the executive would enhance performance. Thus, the CMD file generator block for development platform can be conducted into the memory settings [17].

3.1.2. TLC and RTW. Target language compiler (TLC) is a Matlab program that uses syntax. Developers using the RTW tool can use the TLC to create self-designed C syntax language code by adding to the executive after the RTWgenerated $\mathrm{C}$ language code or design. The use the S-function in the input and output of the set can design its own system for $\mathrm{C}$ programming and create Simulink objects in the box to use; however, RTW is only responsible for producing the $\mathrm{C}$ language program yards. It will not check the correct use of grammar; thus, performing actions or debugging code requires conducting $\mathrm{C}$ into the editor. Moreover, in the design of TLC, all of the program features in metropolis are the function of the type, as shown in Figure 4. Thus, the designer can use the RTW to generate the required developer as long as the $C$ program is appropriately used together with the TLC syntax. The source code, TLC, and RTW program application flowchart is shown in Figure 5 [18].

\section{Simulation Results of the Proposed Systems}

The reference model specifies a number of parameters that can be found in Table 1 .

In this section, the overall performance of the proposed systems in terms of measured BER versus the link overall SNR is discussed for several user profiles and channel profiles. The simulation of the proposed DMWT-OFDM, as compared with DWT-OFDM and FFT-OFDM (with cyclic prefix $1 / 8$ in case OFDM-FFT only) system on multicore 


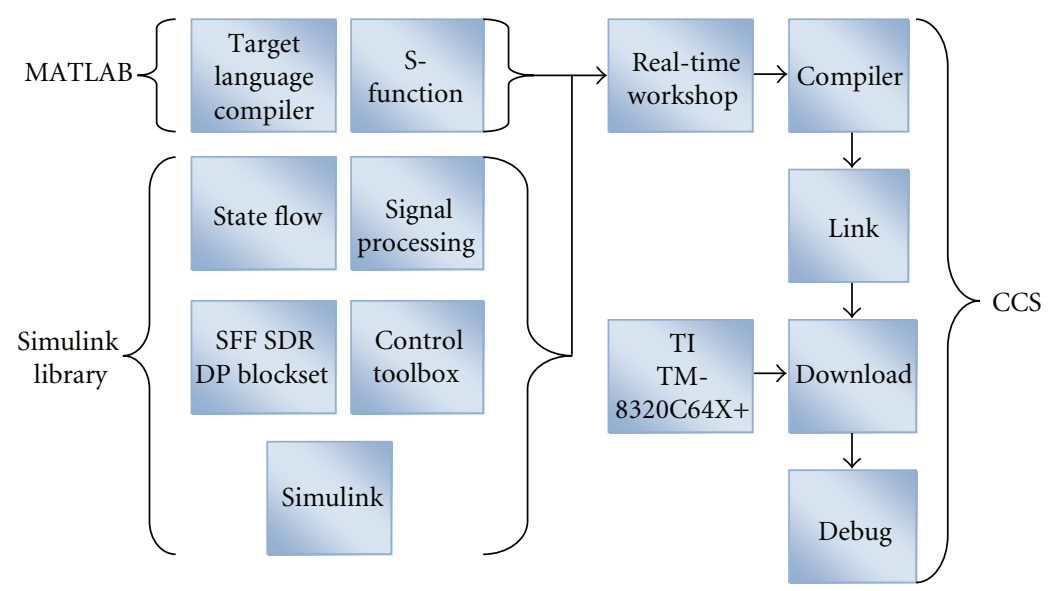

FIgURE 3: Schematic diagram of the system workflow actions [17].

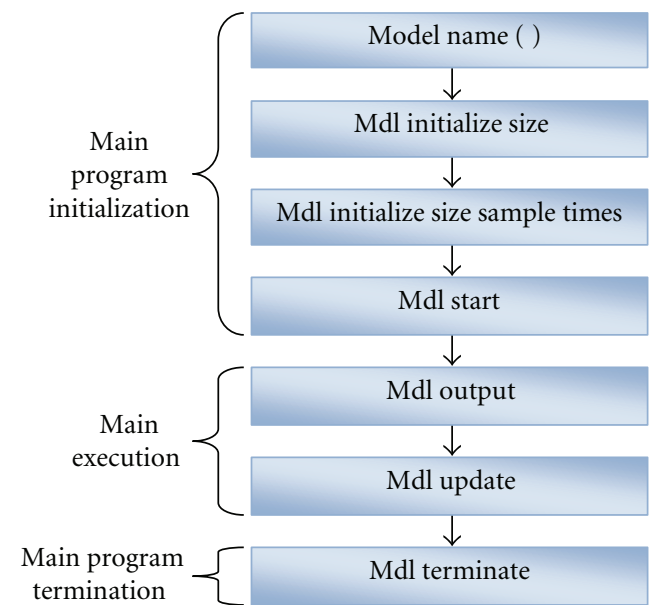

Figure 4: Target language compiler grammatical structure [18].

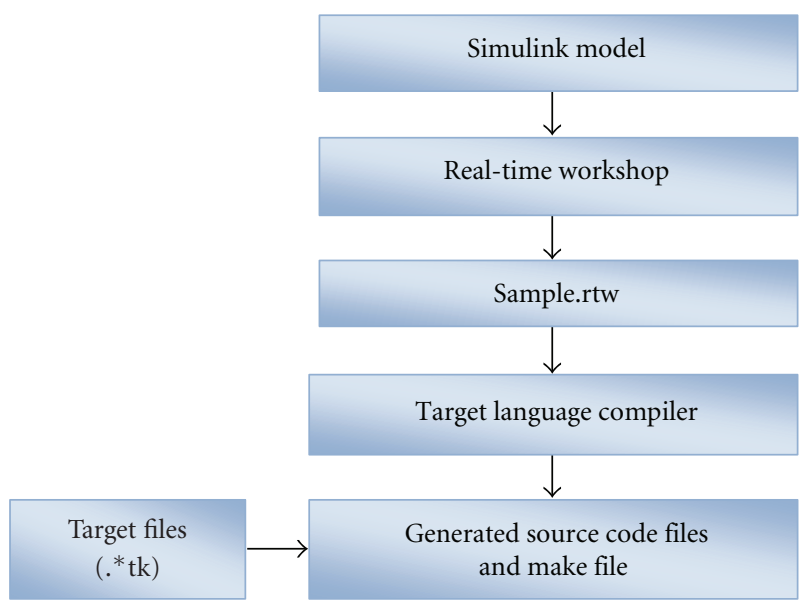

FIgURe 5: TLC and the RTW program application flowchart. software-defined radio platform, is achieved. The BER performance of the OFDM system in different channel models is also considered.

4.1. Performance of AWGN Channel. In this section, the results of the simulation for the proposed DMWT-OFDM system are shown. The results obtained for this case are depicted in Figure 6, which illustrates the BER performance of DMWT-OFDM in AWGN channel. It clearly shows that the DMWT-OFDM is much better than the two previous systems FFT-OFDM and DWT-OFDM. This reflects the fact that the orthogonal base of the multiwavelets is more significant than the orthogonal bases used in FFT-OFDM and DWT-OFDM.

4.2. AWGN Plus Multipath Channel Performance. For this general channel scenario, all ITU profiles are presented in [19]. In the next sections, the relevant results are discussed.

4.2.1. Indoor Channel A. The indoor location user is a fixed subscriber, subsequently its Doppler spread is null. Profile A has shorter delay spread when compared to profile B.
Profile A replicates rural macrocellular surroundings in this scenario and the results obtained were encouraging. From Figure 7 it can be seen that for BER $=10^{-3}$ the SNR required for DMWT-OFDM is approximately $10.2 \mathrm{~dB}$. In DWT-OFDM the SNR approximately $11 \mathrm{~dB}$ and for FFTOFDM approximately $17.5 \mathrm{~dB}$. Figure 7 clearly illustrates that the DMWT-OFDM significantly outperforms other two systems for this channel model.

4.2.2. Indoor Channel B. In this simulation profile some important results were obtained. Recall that the profile of channel $\mathrm{B}$ has a bigger time delay spread than the profile of channel A; more than twice to be more quantitative. This factor plays a huge role in the systems' performances. Observing Figure 8, the BER performance of DMWT-OFDM is better also than the two systems. The DMWT-OFDM had a BER performance of $10^{-3}$ approximately $14 \mathrm{~dB}$. The DWTOFDM had the same BER performance at $16.8 \mathrm{~dB}$, while the OFDM-FFT had a BER performance approximately $25.2 \mathrm{~dB}$. From these results it can be concluded that the DMWTOFDM is more significant than the two systems, based of DWT and FFT in the channel that have been assumed. 


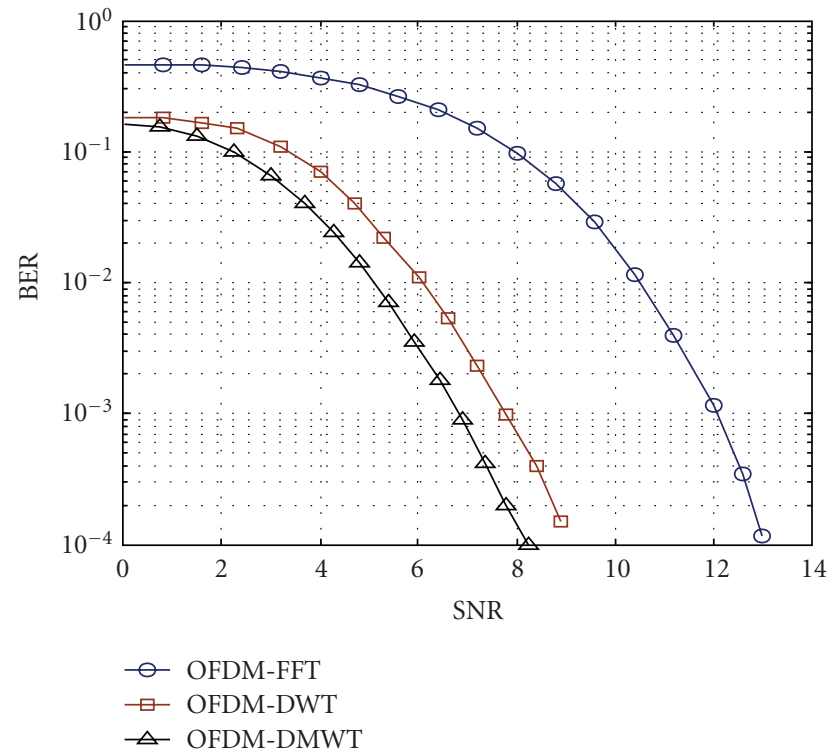

FIGURE 6: BER performance of WiMAX DMWT-OFDM in AWGN channel model.

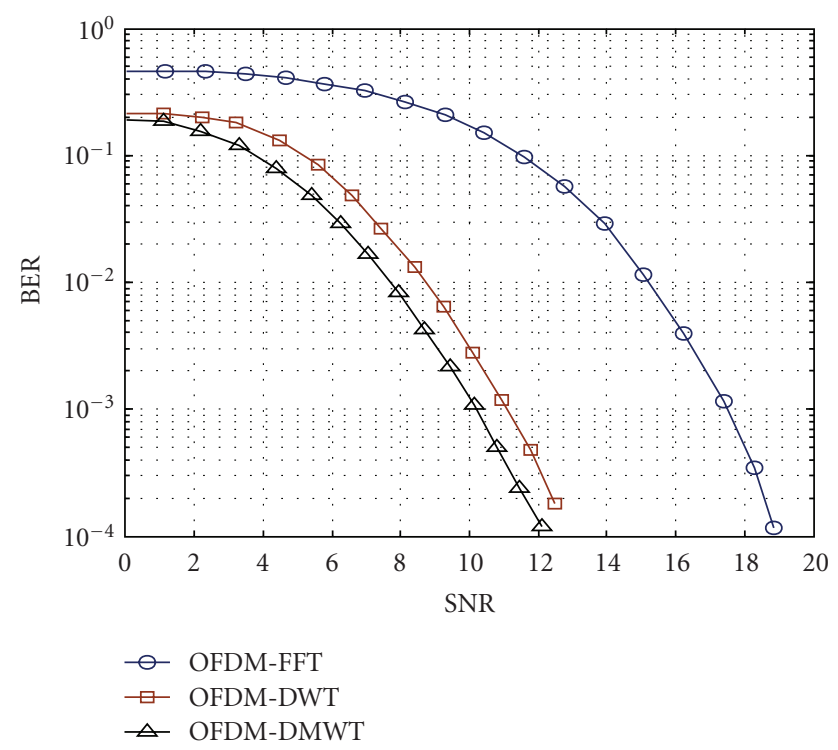

FIGURE 7: BER performance of WiMAX DMWT-OFDM in AWGN plus multipath Indoor channel A.

4.2.3. Pedestrian Channel A. In the pedestrian profile, two different situations were considered: a moving and a stationary person. These results are depicted in Figures 9 and 10. From Figure 9 represents the case stationary person. It can be seen that for BER $=10^{-3}$ the SNR required for DMWT-OFDM was approximately $11.95 \mathrm{~dB}$, approximately $13.3 \mathrm{~dB}$ for DWT-OFDM and approximately $17.4 \mathrm{~dB}$ for FFT-OFDM. Figure 10 presents the case of a moving person. It can also be seen that for $\mathrm{BER}=10^{-3}$ the SNR required for DMWT-OFDM is approximately $14 \mathrm{~dB}$, approximately $16.5 \mathrm{~dB}$ for DWT-OFDM and approximately

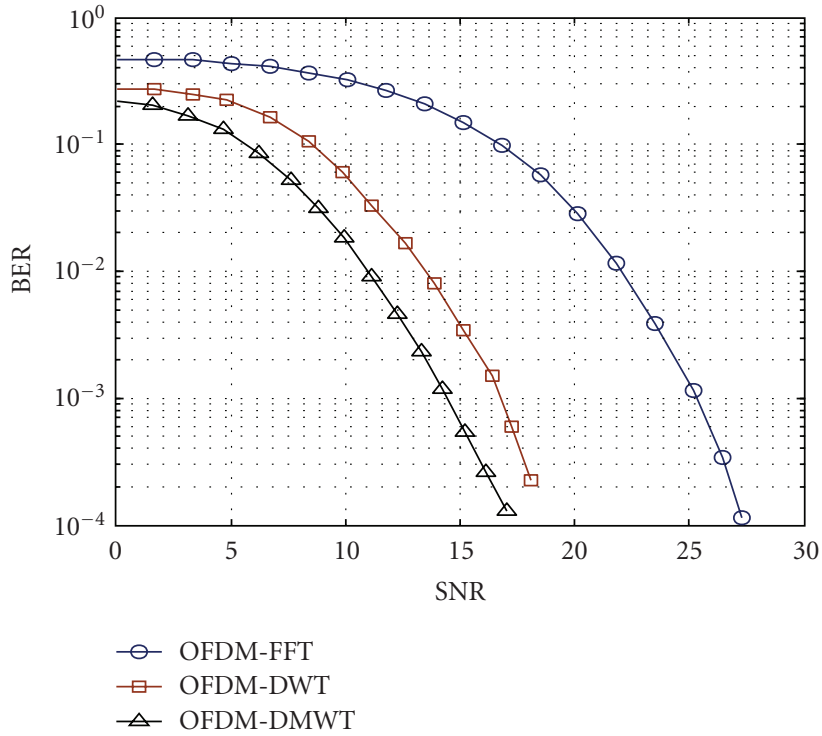

Figure 8: BER performance of WiMAX DMWT-OFDM in AWGN plus Multipath Indoor Channel B.

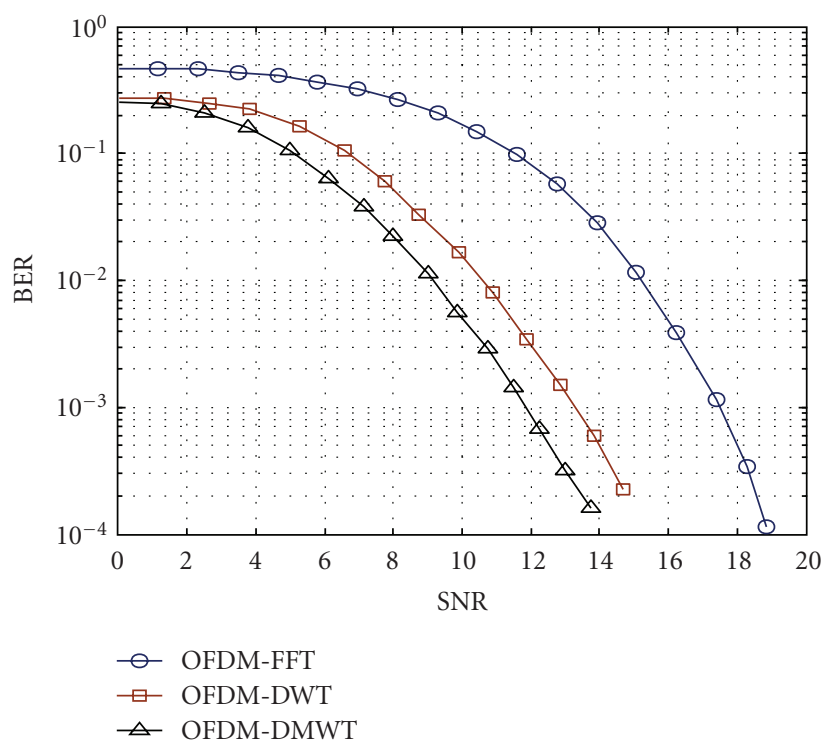

Figure 9: BER performance of WiMAX DMWT-OFDM in AWGN and multipath stationary pedestrian A channel.

$21.85 \mathrm{~dB}$ for FFT-OFDM. Figures 10 and 11 clearly illustrates that the DMWT-OFDM significantly outperforms other two systems for this channel model.

4.2.4. Pedestrian Channel B. Using the same methodology as in the preceding section, simulations for both active and stationary pedestrians were carried out. The results for the case of the stationary pedestrians $\mathrm{B}$ channels are depicted in Figure 12 which shows that for $B E R=10^{-3}$ the SNR required for DMWT-OFDM is approximately $22.3 \mathrm{~dB}$, approximately $23.95 \mathrm{~dB}$ for DWT-OFDM and approximately $26 \mathrm{~dB}$ for 
TABLE 2: Compare between the results.

\begin{tabular}{llccc}
\hline & Channel For BER $=10^{-3}$ & OFDM-FFT dB & OFDM-DWT dB & OFDM-DMWT dB \\
\hline & \multicolumn{1}{c}{ AWGN } & 12 & 7.8 & 11 \\
\hline & Indoor channel A & 17.5 & 17 & 10.3 \\
\hline & Indoor channel B & 25 & 17 & 14.5 \\
\hline \multirow{2}{*}{ Pedestrian Channel A } & AWGN and multipath stopped pedestrian A & 17.2 & 13.25 & 16 \\
& AWGN and multipath active pedestrian A & 22 & 24 & 14 \\
\hline \multirow{2}{*}{ Pedestrian Channel B } & AWGN and multipath stopped pedestrian B & 30 & 37 & 22 \\
& AWGN and multipath active pedestrian B & Poor & 33.2 \\
\hline
\end{tabular}

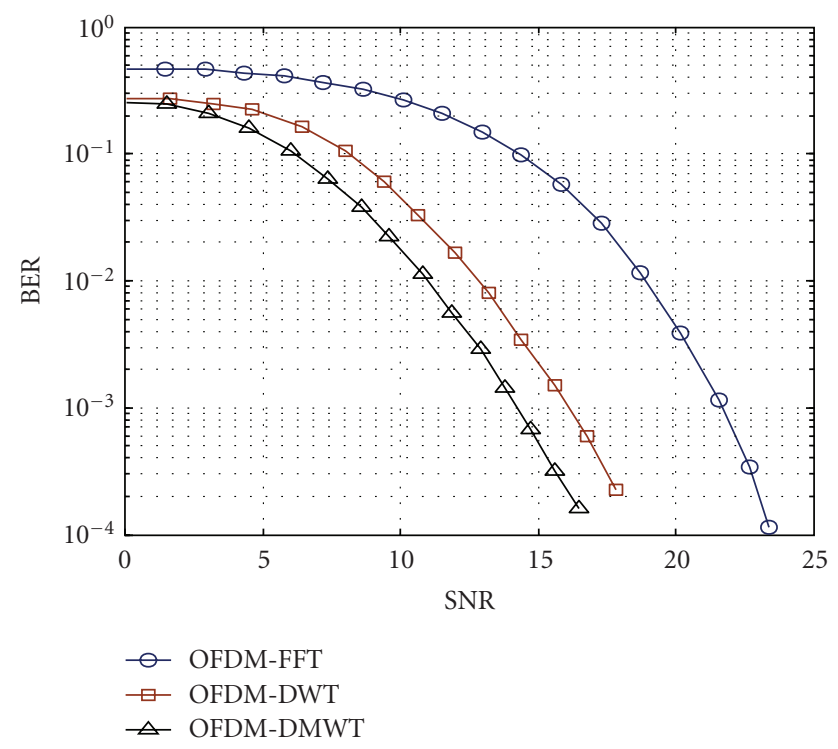

FIGURE 10: BER performance of WiMAX DMWT-OFDM in AWGN and multipath active pedestrian A channel.

FFT-OFDM. The result in active pedestrians is depicted in Figure 12 and it can be seen that for BER $=10^{-3}$ the SNR required for DMWT-OFDM is approximately $31.24 \mathrm{~dB}$, approximately $36.5 \mathrm{~dB}$ for DWT-OFDM and approximately clearly showed overall poor performance for FFT-OFDM. Figures 11 and 12 clearly show that the DMWT-OFDM significantly outperforms the other two systems for this channel model.

A number of important results can be taken from Table 2. In this simulation, in most scenarios, for DMWT-OFDM system is best from the DWT-OFDM system and FFT-OFDM system.

\section{Conclusion}

The DSP of the SFF SDR development platform are completely integrated into the model-based design flow, which integrates Matlab, Simulink, and the Real-Time Workshop from MathWorks. The SFF SCA development platform optional package allowed SCA waveform development and implementation. The key contribution of this paper is the implementation of the IEEE 802.16d PHY-layer-based the DMWT-OFDM structure on the SFF SDR development

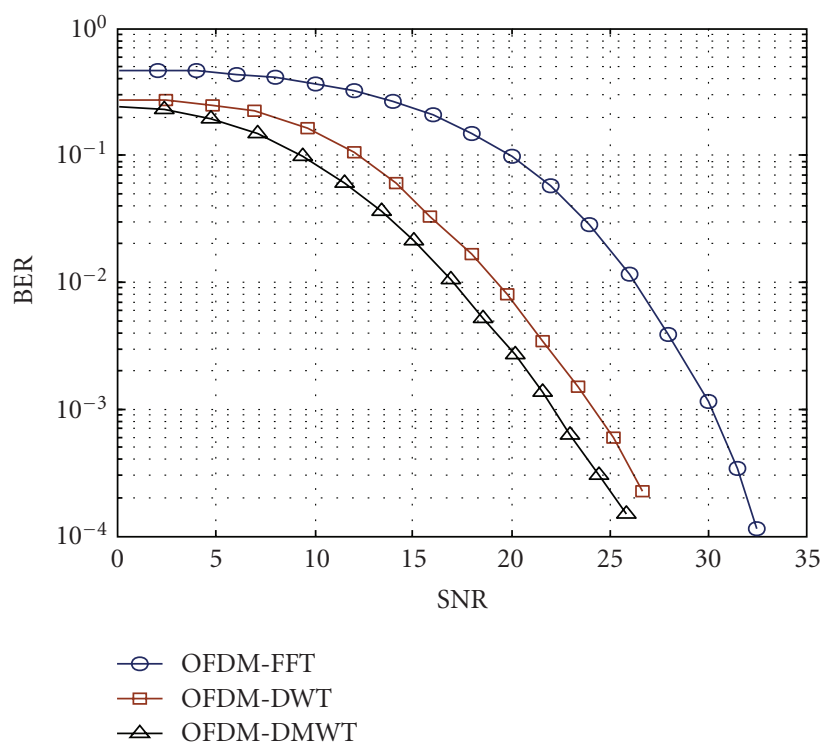

FIGURE 11: BER performance of WiMAX DMWT-OFDM in AWGN and multipath stationary pedestrian B channel.

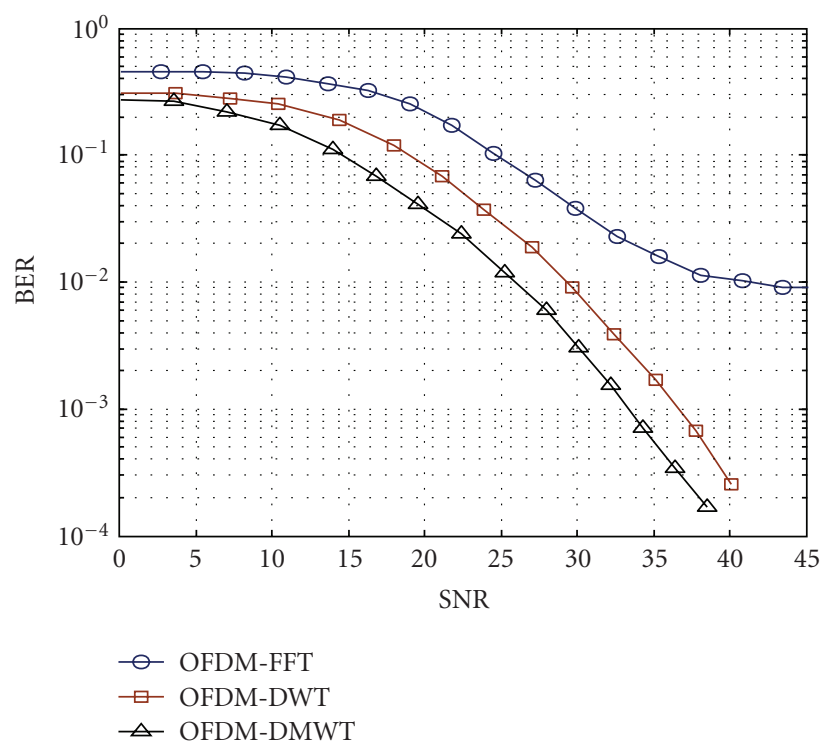

FIgure 12: BER performance of WiMAX DMWT-OFDM Performance in AWGN and multipath active pedestrian B channel. 
platform, which was proposed, simulated, and tested. These tests were carried out to verify its successful operation and possibility of implementation. It can be concluded that this structure achieves much lower bit error rates assuming reasonable choice of the bases function and method of computation. In AWGN and other channels, Simulations proved that the proposed design achieved much lower bit error rates and better performance than FFT-OFDM and DWT-OFDM assuming reasonable choice of the bases function and method of computations. The proposed DMWTOFDM system is robust for multipath channels and does not require cyclically prefixed guard interval, which means that it obtains higher spectral efficiency than conventional OFDMand OFDM based wavelet, therefore, this structure can be considered an alternative to the conventional OFDM, and can be used at high transmission rates.

\section{Acknowledgments}

The authors would like to thank the School of Electrical and Electronic Engineering, USM, and the MOSTI Secretariat, Ministry of Science, Technology, and Innovation of Malaysia, e-Science fund: 01-01-05-SF0239, for sponsoring this paper.

\section{References}

[1] IEEE 802.16, IEEE standard for local and metropolitan area networks-part 16: air interface for fixed broadband wireless access systems, 2004.

[2] WIMAX Forum, "Documentation, technlogy whitepapers," 2010, http://www.wimaxforum.org/resources/documents.

[3] J. G. Andrews, A. Ghosh, and R. Muhamed, Fundamentals to WIMAX Understanding Broadband Wireless Networking, Prentice-Hall, 2007.

[4] A. Ghosh, D. R. Wolter, J. G. Andrews, and R. Chen, "Broadband wireless access with WiMax/802.16: current performance benchmarks, and future potential," IEEE Communications Magazine, vol. 43, no. 2, pp. 129-136, 2005.

[5] A. Graps, "Introduction to wavelets," IEEE Computational Science \& Engineering, vol. 2, no. 2, pp. 50-61, 1995.

[6] A. M. Reza, "From fourier transform to wavelet transform, basic concepts," White Paper 27, Spire Laboratory, University of Wisconsin-Milwaukee, 1999.

[7] B. G. Negash and H. Nikookar, "Wavelet-based multicarrier transmission over multipath wireless channels," Electronics Letters, vol. 36, no. 21, pp. 1787-1788, 2000.

[8] B. G. Negash and H. Nikookar, "Wavelet based OFDM for wireless channels," in Proceedings of the Vehicular Technology Conference, International Research Center for Telecommunications-Transmission and Radar, Faculty of Information Technology and Systems, Delft University of Technology, 2001.

[9] G. W. Wornell and A. V. Oppenheim, "Wavelet-based representations for a class of self-similar signals with application to fractal modulation," IEEE Transactions on Information Theory, vol. 38, no. 2, pp. 785-800, 1992.

[10] M. J. Manglani and A. E. Bell, "Wavelet modulation in Gaussian and Rayleigh fading channels," in Proceedings of the IEEE Military Communications Conference (MILCOM '01), Electrical Engineering, Virginia Polytechnic Institute and State University, McLean, Va, USA, October, 2001.
[11] M. A. Kadhim and W. Ismail, "Implementation of wimax IEEE802. 16d baseband transceiver based wavelet OFDM on multi-core software-defined radio platform," European Journal of Scientific Research, vol. 42, no. 2, pp. 303-313, 2010.

[12] V. Strela, P. N. Heller, G. Strang, P. Topiwala, and C. Heil, "The application of multiwavelet filterbanks to image processing," IEEE Transactions on Image Processing, vol. 8, no. 4, pp. 548$563,1999$.

[13] M. B. Martin, Applications of multiwavelets to image compression, M.S. thesis, Electrical Engineering Department, Virginia Polytechnic Institute and State University (Virginia Tech), Blacksburg, Va, USA, 1999.

[14] T. J. Yew, Multiwavelets and scalable video compression, Ph.D. thesis, Department of Electrical and Computer Engineering, National University of Singapore, 2002.

[15] A. H. Kattoush, W. A. Mahmoud, and S. Nihad, "The performance of multiwavelets based OFDM system under different channel conditions," Digital Signal Processing, vol. 20, no. 2, pp. 472-482, 2009.

[16] J. S. Geronimo, D. P. Hardin, and P. R. Massopust, "Fractal functions and wavelet expansions based on several scaling functions," Journal of Approximation Theory, vol. 78, no. 3, pp. 373-401, 1994.

[17] Texas Instruments Incorporated. TMD SFF SDR DP, 2009, www.ti.com/sdr.

[18] MathWorks, Math Works express, Target Language Compiler, 2002.

[19] International Telecommunication Union, Recommendation, ITU-R M.1225, Guidelines for Evaluation of Radio Transmission Technologies for IMT-2000, 2002. 

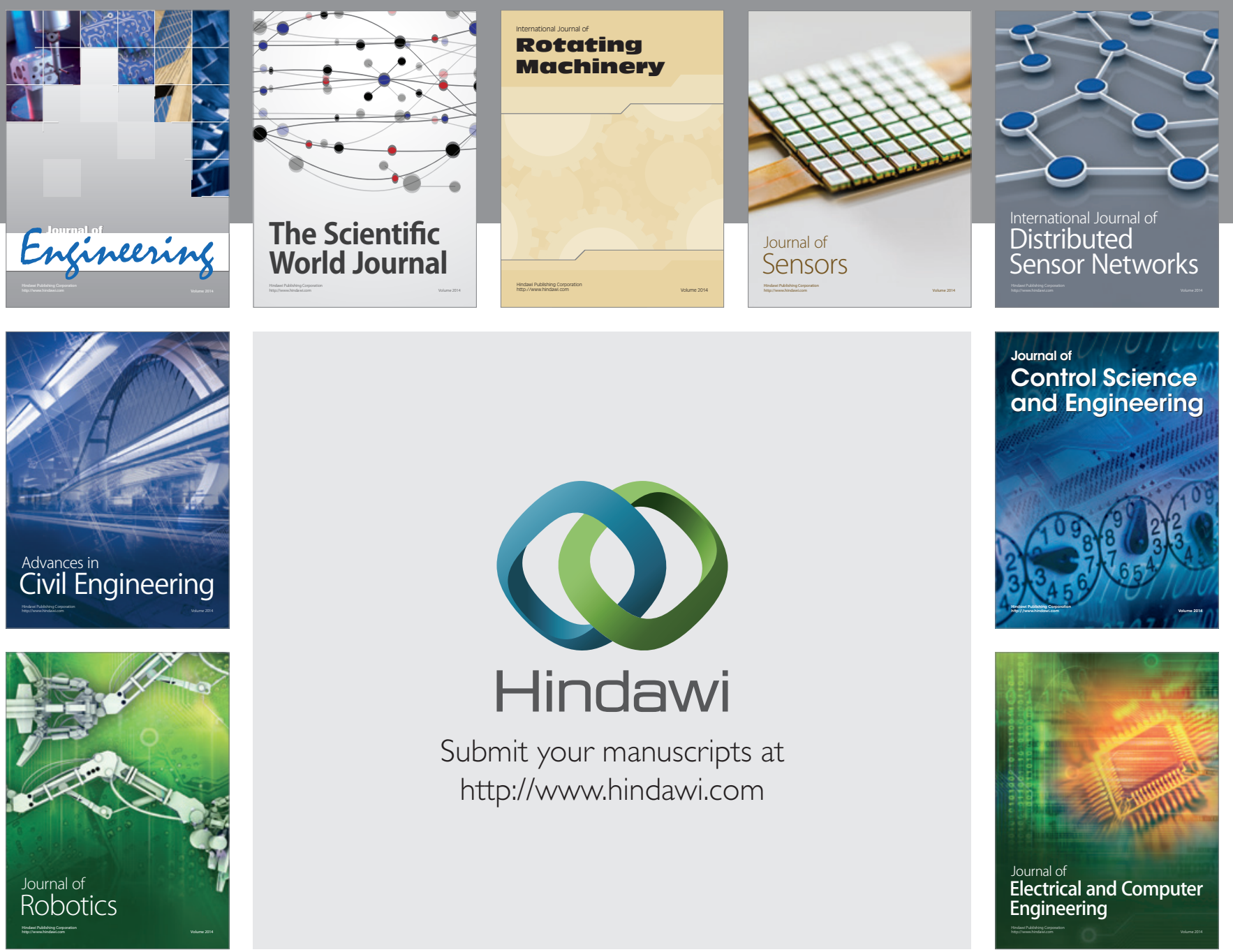

Submit your manuscripts at

http://www.hindawi.com
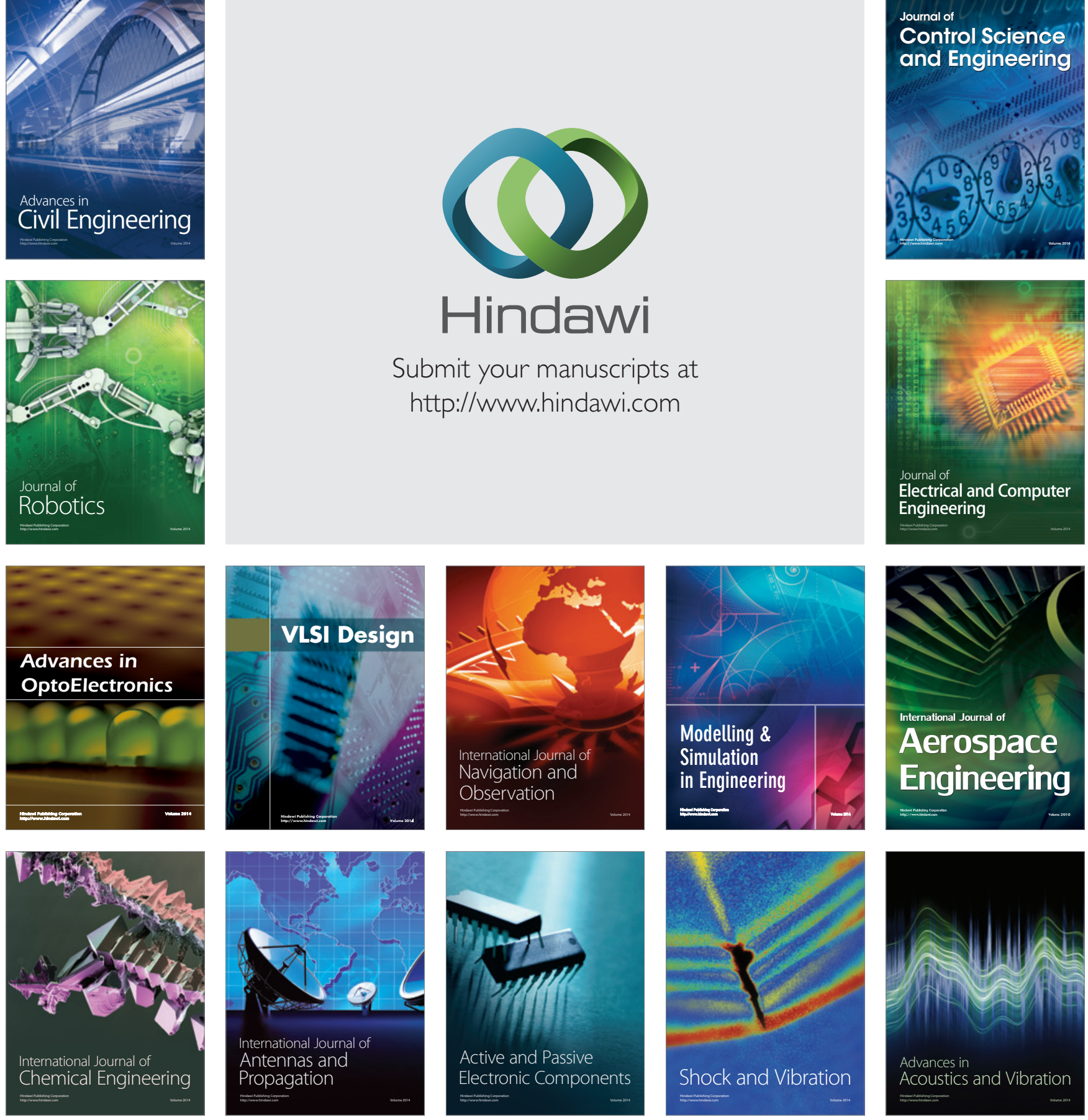